\title{
ELABORAÇÃo DE PRÁTICAS DE GESTÃo DE CUSTOS E PRECIFICAÇÃo DE FRETES PARA PEQUENOS TRANSPORTADORES RODOVIÁRIOS DE CARGA
}

\author{
ELABORATION OF COST MANAGEMENT PRACTICES AND FREIGHT \\ PRECISIONING FOR SMALL CARGO ROAD TRANSPORTERS
}

\author{
Mauro Leite Junior - mauroleitejunior@gmail.com \\ Marco Antonio Alves de Souza Junior - marco_jr90@ hotmail.com \\ Faculdade de Tecnologia de Taquaritinga (FATEC) -SP -Brasil
}

DOI: 10.31510/infa.v16i2.628

\begin{abstract}
RESUMO
O objetivo deste trabalho é a apuração de todos os custos envolvidos, considerando a realidade das operações de transporte de carga rodoviário no Brasil, a fim de apresentar a implementação da Gestão de Custos e Precificação de fretes do segmento rodoviário de cargas. A pesquisa desenvolvida foi aplicada a um pequeno prestador de serviços no segmento mencionado, com resultados no que tange as operações e aspectos financeiros e econômicos considerados satisfatórios diante da gestão receita x despesas. Para tanto, a metodologia utilizada seguiu-se numa abordagem qualitativa, descritiva e exploratória, de revisão bibliográfica em autores consagrados na área, além de pesquisas em artigos científicos no Scielo e Google Acadêmico e revistas especializadas pela internet. Conclui-se que em linhas gerais, o trabalho ora desenvolvido pode proporcionar as partes interessadas uma gestão robusta e adequada as praticas alinhadas ao cenário de transporte rodoviário de cargas.
\end{abstract}

Palavras-chave: Gestão de Custos e Precificação. Prestador de Serviços. Cargas.

\begin{abstract}
The objective of this work is to calculate all the costs involved, taking into account the reality of road freight transport operations in Brazil, in order to present the implementation of Cost Management and Pricing of freight of the cargo road segment. The research developed was applied to a small service provider in the aforementioned segment, with results regarding the operations and financial and economic aspects considered satisfactory before the management revenue $x$ expenses. For that, the methodology used was followed by a qualitative, descriptive and exploratory approach, bibliographical review by renowned authors in the area, as well as research in scientific articles in Scielo and Google Scholar and specialized magazines on the internet. It is concluded that, in general terms, the work developed can provide stakeholders with a robust management and adequate practices aligned the scenario of road freight transport.
\end{abstract}

Keyboards: Cost Management and Pricing. Service Provider. Loads. 


\section{INTRODUÇÃO}

O presente trabalho tem por objetivo a apuração de todos os custos envolvidos, considerando a realidade das operações de transporte de carga rodoviário no Brasil, a fim de apresentar a implementação da Gestão de Custos e Precificação de fretes do segmento rodoviário de cargas, que segundo Bruni (2003), as classificações de custos tendem a sofrer variâncias entre as empresas.

A pesquisa desenvolvida foi aplicada a um pequeno prestador de serviços no segmento mencionado, com resultados considerados satisfatórios no que diz respeito à receita $\mathrm{x}$ despesas. Para o controle e análise de custos, preços e valores foram elaboradas planilhas em Excel, levando-se em consideração, como um todo para o gerenciamento dos resultados: a Quilometragem desenvolvida, Tipo de tráfego, Tipo de via, Região, Porte do veículo e Desequilíbrio de fluxos, além dos custos associados aos encargos salariais dos motoristas e/ou colaboradores em geral.

Este estudo se justifica na medida em que pode auxiliar em formar o preço somente após levantar todos os valores que compõem o custo de transporte de cargas, ressaltando que esses cálculos devem ser feitos regularmente, pois os custos podem ter uma grande variação. Concomitantemente, é importante continuar acompanhando os serviços oferecidos e preços praticados pela concorrência, para se manter competitivo no mercado, tais informações propiciarão uma avaliação mais objetiva e a melhor negociação com os fornecedores.

Nesse trabalho, buscou-se responder a questão problemática: Como gerir custos de forma eficiente a ponto de permitir análises críticas, sustentando tomadas de decisões assertivas e precificações nas prestações de serviços no transporte rodoviário de carga?

Para tanto, a metodologia utilizada segue-se numa abordagem qualitativa, descritiva e exploratória, de revisão bibliográfica em autores consagrados na área, além de pesquisas em artigos científicos no Scielo e Google Acadêmico e revistas especializadas pela internet.

\section{FUNDAMENTAÇÃO TEÓRICA}

De acordo com Valente et al. (2014) há um atraso no desenvolvimento da nação, causado pela precariedade de uma matriz de transportes eficiente. Na contramão dessa precariedade estão os países mais ricos do mundo, comprovado intrinsecamente pela ralação ao 
tamanho do PIB. O escoamento de cargas no Brasil indica uma desconexão absoluta a multimodalidade de transportes. Considerando esse fator, o modal rodoviário incide na grande parte das movimentações e os custos empregados. As movimentações que, segundo Valente et al. (2014) chega a 1.100 barris $^{1}$ de petróleo/dia, indica por si só, a necessidade de uma alta acurácia na gestão de custos associados ao transporte rodoviário de cargas.

Segundo Bruni (2003) entende-se custos como sendo providências monetárias dos sacrifícios assumidos pelas empresas para alcançarem seus objetivos econômicos. Ainda segundo o mesmo, compete a administração financeira a importante análise de custos, preços e valores.

De forma geral, a gestão e o controle dos custos são fundamentais para a saúde financeira da empresa que, segundo Barbosa (2014), a gestão dos custos tem desenvolvido práticas inovadoras e relevantes para suportar a competitividade, bem como, o crescimento no setor de serviços.

Para Valente et al. (2014), a apuração eficaz de custos possibilita tomada de decisões mais conclusivas. De forma geral, o controle e apuração dos custos operacionais devem ser feitos concomitantemente, aos serviços prestados e, considerando o cenário extremamente competitivo, a alimentação dos dados deve ocorrer de forma fidedigna, assim como as análises de forma crítica. Possuir bons conhecimentos acerca dos custos permite converter informações em decisões acertadas. (VALENTE et al., 2014).

\footnotetext{
${ }^{1}$ Equivale em torno de 159,987 litros.
} 
Figura 1 - Custo - Processamento- Decisões

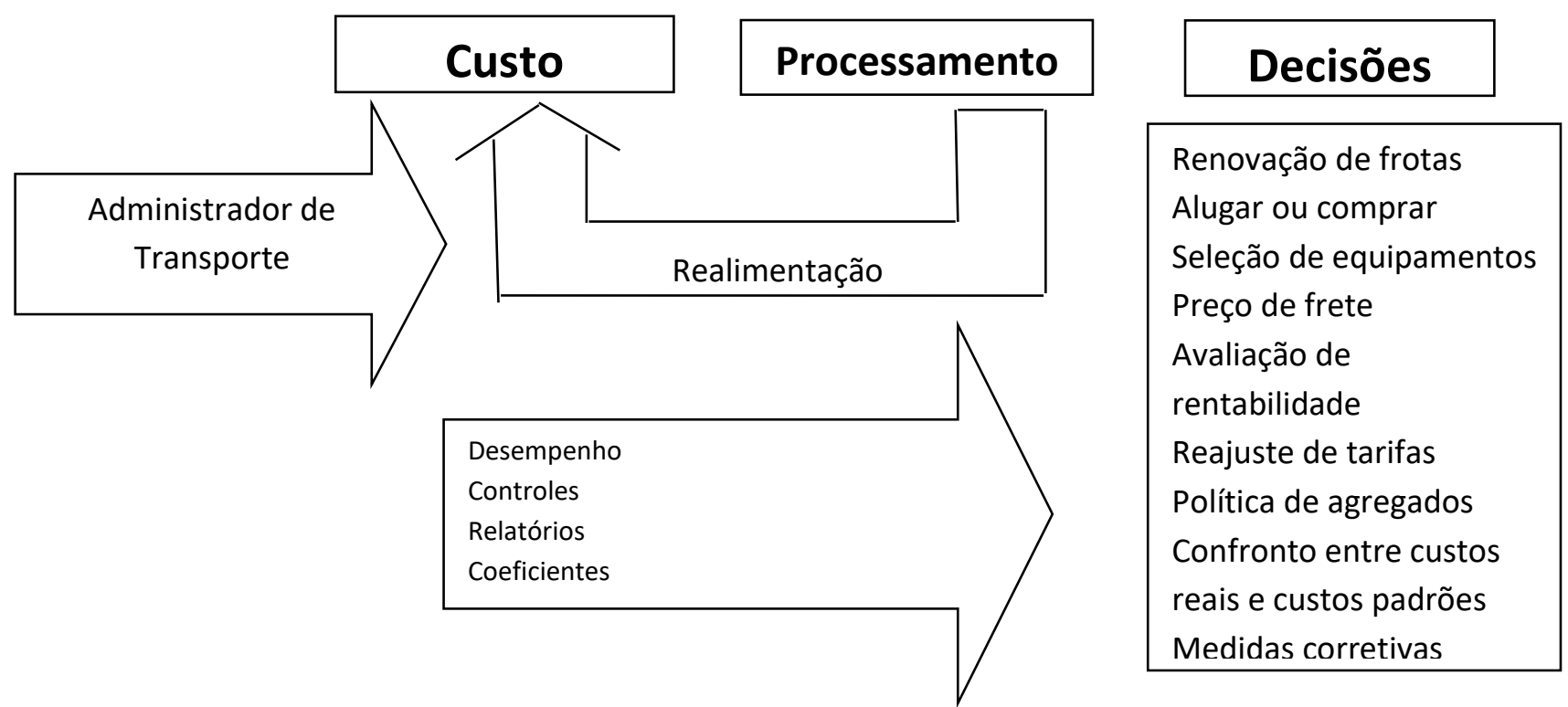

Fonte: Adaptação Valente et al (2014).

$\mathrm{Na}$ aplicação da gestão de custos associam-se às receitas, custos e despesas do pequeno prestador de serviços, portanto, todos os custos estão intrinsecamente relacionados às operações logísticas, especificamente, de transporte rodoviário. As receitas, custos e despesas tendem a sofrer alterações, mediante oscilação de demanda na prestação de serviços e, ainda há de se considerar variações de custos, seja unitário ou total, mediante ao comportamento do volume de produção / operação ofertados. (BRUNI, 2003).

Os fatores que determinam a variações nos custos são: Quilometragem desenvolvida, Tipo de tráfego, Tipo de via, Região, Porte do veículo e desequilíbrio de fluxos. (VALENTE et al., 2014).

Gerir custos é de fundamental importância na gestão de qualquer negócio, independente do porte e segmento e, tão importante quanto à gestão propriamente dita, é a correta forma de se classificar cada um dos custos associados à produção e/ou prestação de serviços.

Para se permitir uma análise econômica e conclusiva, devem-se distinguir os custos de produção, conforme abaixo:

- Custos Diretos: Relacionado diretamente na prestação de serviços;

- Custos Fixos: Custos que não variam, independentemente do volume de serviço prestado;

- Custos Variáveis: Variam de acordo com o volume apresentado na prestação de serviços. (VALENTE et al., 2014). 
Compreender o comportamento dos custos em resposta à mudança dos níveis de atividade econômica é de grande importância para a tomada de decisões dentro da empresa para atingir objetivos estratégicos, com diversos instrumentos de gestão, tais como, análise de margem de contribuição, ponto de equilíbrio, impulsionamento operacional e a margem de segurança. (ATKINSON et al., 2008).

Diante de um cenário extremamente competitivo e com concorrências a níveis extremos, o transporte de cargas rodoviária no Brasil, regulamentado pela Agência Nacional de Transporte Terrestre (ANTT) exige uma apuração de custos e despesas de forma refinada. Análises mais robustas acerca dos custos de operação na prestação de serviços, bem como uma precificação que permita uma margem de contribuição minimamente aceitável ao contratado, indicarão de forma clara quais são as estratégias utilizadas para um equilíbrio na gestão dos custos. (ANTT, 2018). Na falta de uma matriz de transporte melhor distribuída (Figura 2), o modal rodoviário é aquele que mais se destaca no desequilibrado cenário entre oferta e demanda do transporte de cargas no Brasil. Os modais de transporte aqui mencionados são:

- Modal rodoviário: Transporte realizado por meio de rodovias (vias pavimentadas) e estradas (vias não pavimentadas);

- Modal ferroviário: Transporte realizado por meio de ferrovias;

- Modal aéreo: Transporte realizado utilizando o espaço aéreo;

- Modal aquaviário: Transporte realizado por meio de águas (Mares, Rios etc.);

- Modal dutoviário: Transporte realizado por meio de dutos

Figura 2 - Percentual de participação dos modais de transporte

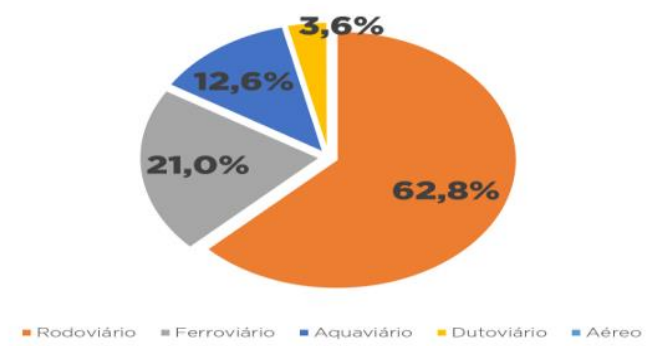

Fonte: Adaptada Fórum Internacional de Supply Chain - ILOS (2016)².

Em um processo natural na massificação de veículos de carga, veio a ser contemplado com oferta e consumo de recursos nunca vistos anteriormente. A partir desse

\footnotetext{
2 Cf. Transportes de cargas e a encruzilhada do Brasil para o futuro, por Alexandre Lobo. Disponível em:> http://www.ilos.com.br/web/tag/transporte-de-carga/Acesso em 13 de maio de 2018.
} 
ponto, o modal rodoviário brasileiro passa a ser utilizado em percentuais cada vez maiores, conforme visto na Figura 2. (MARTINS, 2008).

O desequilíbrio entre "oferta $\mathrm{x}$ demanda" (Figura 3), onde "oferta" representa a disponibilidade de recursos para o transporte (Veículos) e a "demanda" está vinculada a quantidade de serviços desejados pelos embarcadores. (CNT, 2018).

Figura 3 - Gráfica demanda na prestação de serviços rodoviário

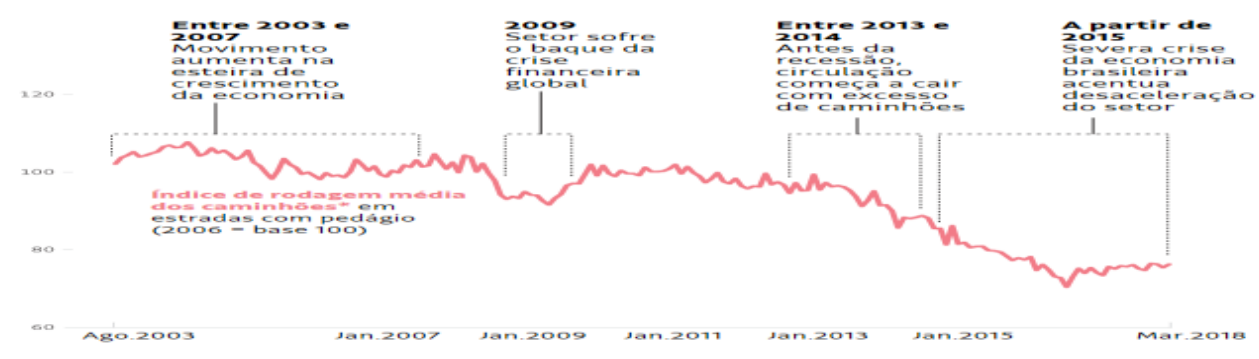

Fonte: Confederação Nacional do Transporte (2018) ${ }^{*}$

Conforme estudo entre 137 países, realizada pela Word Economic Forum (WEF, 2018), o Brasil ocupa posições dianteiras nos componentes de infraestrutura logística.

Figura 4 - Mapa Estratégico da Indústria 2018-2022

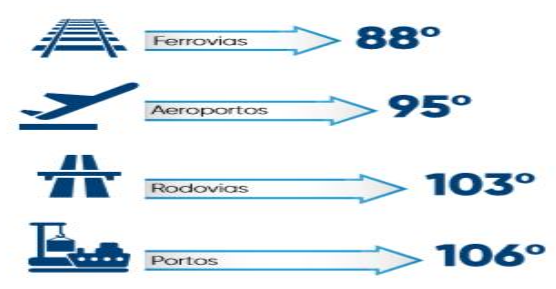

Fonte: Confederação Nacional das Indústrias. (WEF, 2018) ${ }^{3}$

Todos esses componentes, reforçam de maneira muito clara a necessidade de apurações de custos de forma ajustada, evitando dessa maneira, potenciais perdas por tomadas de decisões empíricas, ou seja, sem uma base de informações sustentável e confiável. (REIS, 2001).

\footnotetext{
3 Cf. O relatório da competividade global de 2018. World Economic Forun (WEF). Disponível em:> https://www.weforum.org/reports/the-global-competitveness-report-2018. < Acesso em 16 de ago. de 2018.
} 


\section{PROCEDIMENTOS METODOLÓGICOS}

A pesquisa bibliográfica, de acordo com Silva e Menezes (2001, p. 21), é "elaborada a partir de material já publicado, constituído principalmente de livros, artigos de periódicos e atualmente com material disponibilizado na Internet."

Fundamentou-se em uma abordagem qualitativa, descritiva e exploratória, aplicando-se a um pequeno prestador de serviços no segmento transporte rodoviário de carga, a apuração de todos os custos envolvidos, considerando a realidade das operações de transporte de carga rodoviário no Brasil, a fim de apresentar a implementação da Gestão de Custos e Precificação de fretes do segmento rodoviário de cargas.

Para os custos de pedágio, utilizou-se das informações obtidas no site Qualp (2019) ${ }^{4}$. E para o controle e análise de custos, preços e valores foram elaboradas planilhas em Excel, levando-se em consideração, como um todo para o gerenciamento dos resultados: a Quilometragem desenvolvida, Tipo de tráfego, Tipo de via, Região, Porte do veículo e Desequilíbrio de fluxos, além dos custos associados aos encargos salariais dos motoristas e/ou colaboradores em geral.

\section{RESULTADOS E DISCUSSÃO}

\subsection{Planilha dos custos e formação dos preços}

A composição dos custos está associada ao preenchimento dos respectivos campos em branco, referente às planilhas nas tabelas que seguem.

Tabela 1 - Custos Fixos

\section{Custos da empresa}

Salário do motorista

Encargos salariais do motorista

Horas de trabalho mês

Taxa de oportunidade

$\$ /$ mês

Custo de overhead veículo

h.h./mês

$\%$ a.a.

\$/ mês

${ }^{4}$ Cf. Qualp Calcule a sua viagem. Disponível em:> https://qualp.com.br/.> Acesso em 23 de jun. de 2019. 
Fonte: Fuchs ${ }^{5}$, (2016).

A formação dos custos associados aos encargos salariais do eventual(s) motorista(s) e/ou colaboradores em geral, podem ser considerados conforme Tabela 2. Para o exemplo aqui desenvolvido, considerou-se uma jornada de 44 horas semanais. Os valores inseridos são ilustrativos e, servem apenas para melhor didática para assimilação, pois se referem ao ano de 2003. (BRUNI, 2003).

Tabela 2 - Custos de Mão de Obra

\begin{tabular}{|c|c|}
\hline Número de dias por ano & 365 \\
\hline (-) Repousos semanais remunerados & -48 \\
\hline (-) Férias & -30 \\
\hline (-) Feriados em média & -12 \\
\hline (=) Número máximo de dias à disposição & 275 \\
\hline (x) Jornada diária & 7,з33з \\
\hline (=) Número máximo de horas à disposição & 2016,66 \\
\hline \multicolumn{2}{|l|}{ a) Salários } \\
\hline Numero máximo de horas à disposição & $2.016,66$ \\
\hline Valor da hora trabalhada & $R \$ 5,67$ \\
\hline Total de Salários & $\mathrm{R} \$ \mathbf{1 1 . 4 3 9 , 9 6}$ \\
\hline \multicolumn{2}{|l|}{ b) Repousos semanais remunerados } \\
\hline Número de repousos em dias & 48 \\
\hline Jornada Diária & 7,3333 \\
\hline Número de repousos em horas & 352 \\
\hline Valor da hora trabalhada & $R \$ 5,67$ \\
\hline Total de repousos semanais remunerados & R\$ $1.995,83$ \\
\hline \multicolumn{2}{|l|}{ c) Férias } \\
\hline Férias em dias & зо \\
\hline Jornada diária & 7,з3зз \\
\hline Férias em horas & 220 \\
\hline Valor da hora trabalhada & $\mathrm{R} \$ 5,67$ \\
\hline Total de Férias & R\$ $1.247,40$ \\
\hline \multicolumn{2}{|l|}{ d) Adicional constitucional de férias } \\
\hline Porcentual constitucional & $33,33 \%$ \\
\hline Total de férias & $\mathrm{R} \$ 1.247,40$ \\
\hline Total adicional de férias & $\mathrm{R} \$ \mathbf{4 1 5}, 76$ \\
\hline \multicolumn{2}{|l|}{ e) $13^{\circ}$ Salário } \\
\hline 13ㅇe em dias & 30 \\
\hline Jornada diária & 7,3333 \\
\hline $13 \circ$ em horas & 220 \\
\hline Valor da hora trabalhada & $\mathrm{R} \$ 5,67$ \\
\hline Total de 13o salário & $\mathrm{R} \$ \mathbf{1} 1.247,40$ \\
\hline \multicolumn{2}{|l|}{ f) Feriados } \\
\hline Feriados em dias & 12 \\
\hline Jornada diária & 7,3333 \\
\hline Feriados em horas & 88 \\
\hline Valor da hora trabalhada & $\mathrm{R} \$ 5,67$ \\
\hline Total de feriados & R\$ 498,96 \\
\hline \multicolumn{2}{|l|}{ Contribuições Percentuais } \\
\hline $\begin{array}{l}\text { Previdência Social } \\
\text { Fundo de Garantia }\end{array}$ & $\begin{array}{r}20 \% \\
8 \%\end{array}$ \\
\hline $\begin{array}{l}\text { Fundo de Garantia } \\
\text { Seguro (acidentes de trabalho) }\end{array}$ & $\begin{array}{l}8 \% \\
3 \%\end{array}$ \\
\hline Salário (educação) & $2,5 \%$ \\
\hline Sesi ou Sesc & $1,5 \%$ \\
\hline Senai ou Senac & $1 \%$ \\
\hline Incra & $0,2 \%$ \\
\hline Sebrae & $\begin{array}{r}0,6 \% \\
36,8 \%\end{array}$ \\
\hline
\end{tabular}

${ }^{5}$ Cf. FUNDAMENTOS DA LOGÍSTICA E CADEIA DE SUPRIMENTOS - Disciplina MBA em Logística \& Supply Chain Management - 2016 - Fundação Getúlio Vargas. Prof. Dr. Angelo Fuchs. Disponível em:> https://docplayer.com.br/amp/45007085-Mba-logistica-e-supply-chain.html.> Acesso em 16 de ago. de 2018. 


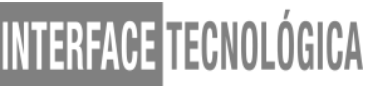

a) Salários

b) Repousos semanais remunerados

c) Férias

d) Adicional Constitucional de férias

e) 13 o Salário

Acréscimo legal de outras contribuições

Total com contribuições

Número de horas trabalhadas por ano

Total geral por hora

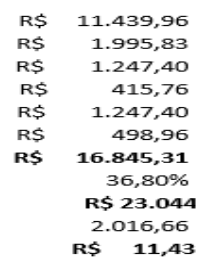

Fonte: Bruni (2003).

A taxa de oportunidade mencionada é o percentual eventual de ganho, caso o investimento aqui realizado fosse aplicado em outra fonte de rendimento, tais como poupança, tesouro e etc. O custo de Overhead é o valor salarial que o colaborador e/ou colaboradores que estão envolvidos com os veículos se dedicam aos equipamentos.

Tabela 3 - Custos Variáveis

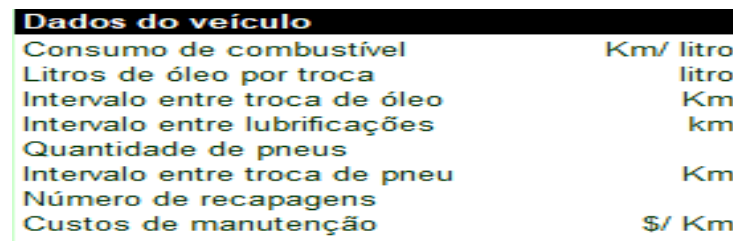

Fonte: Fuchs (2016).

Tabela 4 - Custos de Mercado

\begin{tabular}{|c|c|}
\hline \multicolumn{2}{|l|}{ Dados de mercado } \\
\hline $\begin{array}{l}\text { Valor de aquisição do veículo } \\
\text { Vida útil do veículo }\end{array}$ & meses \\
\hline Valor residual do veículo & $\$$ \\
\hline Preço do óleo & $\$ /$ litro \\
\hline Preço do combustivel & $\$ /$ litro \\
\hline Preço do pneu & $\$$ \\
\hline Preço da recapagem & $\mathrm{RS}$ \\
\hline Rastreadores & \$/Ano \\
\hline Gerenciamento de Risco & \$/Ano \\
\hline Parcela de Financiamento & \$/Ano \\
\hline Preço da lavagem e lubrificação & $\$ / A n o$ \\
\hline IPVA & $\$ /$ Ano \\
\hline Seguro do veículo & \$/An \\
\hline
\end{tabular}

Fonte: Fuchs (2016).

Diante da necessidade de implementação de uma gestão de custos e precificação de fretes, aplicou-se a metodologia ora exposta a um pequeno prestador de serviços do segmento de transporte rodoviário de cargas, localizado no interior do Estado de São Paulo.

O passo inicial consiste em levantar todos os dados financeiros e, portanto, esse passo torna-se fundamental para uma precificação dentro da realidade e do tamanho do negócio. Qualquer situação superestimada ou subestimada em relação aos dados planilhados, pode acarretar em problemas que, ou coloquem em risco a saúde financeira da empresa ou uma percepção de mercado irreal. 


\subsection{Aplicação dos Custos Fixos e Dados de Mercado}

Os dados aqui aplicados são reais e, flexíveis diante da particularidade do transportador. Vale ressaltar que, cada transportador, independentemente do tamanho e características, possuem os valores intrínsecos às suas operações, portanto inerentes aos seus custos operacionais.

Tabela 5 - Custos da Empresa (Preenchida)

$\begin{array}{lrr}\text { Custos da empresa } & \$ / \text { mês } & 4000,00 \\ \text { Pró-Labore } & \text { h.h./mês } & 176 \\ \text { Horas de trabalho/ mês } & \$ / \text { mês } & 800,00 \\ \text { Encargos (Previsencia Social) } & \$ / \text { mês } & 150,00 \\ \text { Contador } & \% \text { a.a. } & 12,0 \% \\ \text { Taxa de oportunidade } & \$ / \text { mês } & \\ \text { Custo de overhead/ veículo } & \end{array}$

Fonte: Fuchs (2016).

Tabela 6 - Dados de Mercado (Preenchido)

\begin{tabular}{lrr}
\hline Dados de mercado & & \\
\hline Valor de aquisição do veículo & $\$$ & $\$ 115.000,00$ \\
Vida útil do veículo & meses & 60 \\
Valor residual do veículo & $\$$ & $\$ 66.250,00$ \\
Preço do óleo & $\$ /$ litro & 8,2 \\
Preço do combustivel & $\$ /$ litro & 3,6 \\
Preço do pneu & $\$$ & 600 \\
Preço da recapagem & $\mathrm{R} \$$ & 360 \\
Preço da lavagem e lubrificação & $\$$ & 0 \\
IPVA & $\$ /$ ano & 2700 \\
Seguro do veículo & $\$ /$ ano & 5000
\end{tabular}

Fonte: Fuchs (2016).

(Obs. Os valores aqui identificados como Dados de mercado compõem aos custos fixos e custos variáveis).

Tabela 7 - Itens de Custo Fixo mês (Preenchido)

\begin{tabular}{|c|c|c|c|}
\hline \multicolumn{4}{|l|}{ Itens de custo fixo } \\
\hline $\begin{array}{l}\text { Depreciação } \\
\text { Remuneração de capital }\end{array}$ & $\begin{array}{l}\text { (\$/ mês) } \\
\text { (\$/ mês) }\end{array}$ & 479,17 & informados \\
\hline Mão de obra & (\$/ mês) & $4.950,12$ & correspondem ao resultado de fórmulas \\
\hline $\begin{array}{l}\text { Gerenciamento de Risco } \\
\text { Licenciamento }\end{array}$ & $\begin{array}{l}\text { (\$/ mês) } \\
\text { (\$/ mês) }\end{array}$ & $\begin{array}{r}132,00 \\
9,20\end{array}$ & coletadas das planilhas Custo da Empresa \\
\hline Seguros (Carga e Veículo) & (\$/ mês) & 416,67 & e Dados de Mercado, ou Tabelas 5 e 6 \\
\hline $\begin{array}{l}\text { Parcela Financiamento } \\
\text { IPVA }\end{array}$ & $\begin{array}{l}\text { (\$/ mês) } \\
(\$ / \text { mês) }\end{array}$ & $\begin{array}{r}2.600,00 \\
225,00\end{array}$ & respectivamente. \\
\hline$\overline{\mathrm{CF}}$ & (\$/ mês) & $8.812,15$ & \\
\hline
\end{tabular}

Fonte: Fuchs (2016).

\subsection{Aplicação dos Custos Variáveis e Dados do Veículo}

A captação dos custos variáveis é de fundamental importância para identificar o quanto há de acréscimo nos custos a cada quilômetro percorrido. 
Tabela 8 - Dados do Veículo (Preenchido)

\begin{tabular}{lrr} 
Dados do veículo & & \\
\hline Consumo de combustível & $\mathrm{Km} /$ litro & 10 \\
Intervalo entre troca de óleo & $\mathrm{Km}$ & 10000 \\
Litros de óleo por troca & litro & 8 \\
Número de pneus & $\mathrm{Km}$ & 4 \\
Intervalo entre troca de pneu & & 240000 \\
Número de recapagens & $\$ / \mathrm{Km}$ & 3 \\
Custos de manutenção & $\mathrm{Km}$ & 0,25 \\
Intervalo entre lubrificações & & 5200
\end{tabular}

Fonte: Fuchs (2016).

Tabela 9 - Itens de Custo variável

\begin{tabular}{llc} 
Itens de custo variável & & \\
\hline Combustivel & $(\$ / \mathrm{Km})$ & 0,360 \\
Óleo & $(\$ / \mathrm{Km})$ & 0,007 \\
Pneu & $(\$ / \mathrm{Km})$ & 0,14 \\
Lubrificação & $(\$ / \mathrm{Km})$ & 0,000 \\
Manutenção & $(\$ / \mathrm{Km})$ & 0,41 \\
\hline \hline Custo variável & $(\$ / \mathrm{Km})$ & 0,921
\end{tabular}

Os valores aqui informados correspondem ao resultado de fórmulas coletadas das planilhas Dados de Veículos e Dados de Mercado, ou tabelas 6 e 8 respectivamente.

Fonte: Fuchs (2016).

\subsection{Aplicando custos dos fretes}

Considerando os valores absorvidos do conjunto das planilhas de custos fixos e variáveis, aplica-se a seguinte dinâmica de configuração nos valores dos fretes:

Exemplo: Frete com total de $350 \mathrm{~km}$.

a) Considera-se em torno de 5 horas de operação, sendo 4 horas em trânsito e 1 hora para carregamento e emissão de nota fiscal.

a.1) O Custo Fixo mensal é R\$ 8.812,15, temos 176 horas de trabalho mensal, portanto, temos $\mathrm{R} \$ 50,07 \mathrm{p} /$ hora

a.2) Considerando apenas os Custos Fixos temos $\mathrm{R} \$ 250,34$

b) Temos um custo variável em torno de $\mathrm{R} \$ 0,92$ por $\mathrm{km}$

b.1) Considerando o Custo Variável, teremos $\mathrm{R} \$ 322,00$

c) A soma dos Custos Fixos e Variáveis contemplam o valor de $\mathrm{R} \$ 572,34$

d) Nesse custo acima mencionado deve-se acrescer os valores de pedágio e Impostos associados ao transporte (ICMS, PIS e CONFINS). 


\section{CONSIDERAÇÕES FINAIS}

Essa gestão, conforme aqui explorada, deve ser controlada de forma minuciosa e, nunca negligenciada, uma vez que o sucesso financeiro da empresa estará intrinsecamente relacionado a seu gerenciamento.

Há dentro do mesmo segmento, empresas com propostas de prestações de serviços, características, bem como, diferentes proporções dimensionais, fazendo-se necessário identificar que a gestão aqui aplicada, contempla um transportador de carga lotação / dedicado (a formação do custo de frete aqui desenvolvido não contempla fracionados).

Sugere-se, portanto, novos estudos para aprofundar a análise, considerando a realidade de outras instituições e suas variantes, a fim de se elaborar novas práticas de gestão de custos e precificação de fretes para pequenos transportadores rodoviários de carga, identificando tendências e estabelecendo estatísticas úteis.

Conclui-se que o estudo foi importante para a microempresa, possibilitando uma verificação de acompanhando dos serviços oferecidos e preços praticados pela concorrência, mantendo-a competitiva no mercado, de posse de informações que propiciarão uma avaliação mais objetiva e a melhor negociação com os fornecedores e uma boa percepção do mercado consumidor desse segmento. Além de exercícios de custos elaborados de forma eficiente, a ponto de permitir investigações críticas, sustentando tomadas de decisões assertivas, garantindo assim, a boa saúde financeira do negócio.

\section{REFERÊNCIAS}

ATKINSON, Anthony A. et al. Contabilidade gerencial. 2. ed. São Paulo: Atlas, 2008.

BARBOSA, C. et al. Gerenciamento de custos em projetos - 5. ed. rev. - Rio de Janeiro: Editora FGV, 2014.

BRASIL. AGENCIA NACIONAL DE TRANSPORTES TERRESTRES - ANTT. Disponível em:>http://www.antt.gov.br/salaImprensa/noticias/arquivos/2018/03/ANTT_lanca_rede_inteli gente_de_mapeamento_logistico.html>. Acesso em: 16 ago. 2018.

BRASIL. CONFEDERAÇÃO NACIONAL DO TRANSPORTE - CNT. Disponível em: > https://www.cnt.org.br/home>. Acesso em: 16 ago. 2018.

BRUNI, A. L. et al. Gestão de Custos e Formação de Preços - 3. ed. rev. - São Paulo: Atlas, 2003. 
CONFEDERAÇÃO NACIONAL DAS INDÚSTRIAS. Mapa estratégico da indústria 2018-2022 / Confederação Nacional da Indústria. - Brasília: CNI, 2018. 209 p.:il.

DOS REIS, N. Regulamentação do transporte rodoviário de cargas no Brasil e no Mundo, 2001. Disponível em: >www.ntcnet.org.br/regtrc>. Acesso em 17 ago. 2018.

FUCHS. Fundamentos da Logística Cadeia de Suprimentos - Disciplina MBA em Logística \& Supply Chain Management FGV-2016

MARTINS, S. Estudo da formação do frete rodoviário e potencial de conflitos em negociações. cadeias do agronegócio Brasileiro, vol. 10, n. 1, pp. 73-87, 2008. Disponível em: > http://revista.dae.ufla.br>. Acesso em: 29 set. 2018.

O RELATÓRIO DA COMPETIVIDADE GLOBAL DE 2018. WORLD ECONOMIC FORUN (WEF). Disponível em: > https://www.weforum.org/reports/the-global-competitveness-report2018>. Acesso em: 16 ago. 2018.

QUALP CALCULE A SUA VIAGEM. Disponível em: > https://qualp.com.br/.> Acesso em: 16 ago. 2018.

RECESSÃO FEZ DESPENCAR NUMERO DE VIAGENS DE CAMINHÃO. Disponível em: $>$ https://www1.folha.uol.com.br/mercado/2018/06/recessao-fez-despencar-numero-deviagens-de-caminhao.shtml>. Acesso em: 04 jun. 2018.

SILVA, E. L. MENEZES, E. M. Metodologia da pesquisa e elaboração de dissertação. 3. ed. Florianópolis: Laboratório de Ensino a Distância da UFSC, 2001.

TRANSPORTES DE CARGAS E A ENCRUZILHADA DO BRASIL PARA O FUTURO, POR ALEXANDRE LOBO. Disponível em: > http://www.ilos.com.br/web/tag/transporte-decarga>. Acesso em: 13 maio 2018.

VALENTE, A. M. et al. Gerenciamento de transporte e frotas - 2. ed. rev. - São Paulo: Cengage Learning, 2014. 